\title{
Oculomotor adaptation to wedge prisms with no part of the body seen
}

\author{
SAMUEL C. MCLAUGHLIN, KENNETH I. RIFKIN AND ROBERT G. WEBSTER
}

When $S$ looks at a visual target through prisms, adaptive shifts in reaching behavior occur even though he sees no part of his body through the prisms. These shifts are caused by a change in the judgment of the direction of gaze (oculomotor change), which in turn is caused by two secondary prismatic effects: (a) asymmetry of the visual display and (b) apparent rotation about a vertical axis of a panel or wall facing S. The "asymmetry" factor contributes $22 \%$ of the total oculomotor change, and the "rotation" effect contributes the remaining 78\%. Oculomotor change is not facilitated by eye-movement activity. The adaptive oculomotor change induces a non-adaptive proprioception change about one-tenth as large as the oculomotor change. These findings are capable of accounting for the previously unexplained results reported by Wooster in 1923, and also for the current controversy about the role of reafferent stimulation in sensorymotor adaptation.

Adaptation to lateral prismatic displacement was described by von Helmholtz in $1867 .{ }^{1}$ Wearing spectacles which contained wedge prisms, he first demonstrated the apparent displacement by looking at objects, then closing his eyes and trying to touch them. He reached incorrectly, the direction of his error being determined by the orientation of the prisms. He then found that he could eliminate the error in either of two ways: by reaching repeatedly for objects with eyes closed, or, "more quickly still," by touching the objects several times while watching his hand through the prisms. Having adapted by either of these techniques, he repeated the initial procedure as a means of demonstrating that adaptation had taken place: "on trying the above experiment again, we shall discover that now we do not miss the objects but feel for them correctly."

Helmholtz went on to investigate the locus of the adaptive effect. He found that, if he first adapted by doing all of the reaching with his right hand, keeping his left hand out of his field of view, the adaptive effect nevertheless transferred fully to his left hand. He concluded that the adaptive change could not be in the judgment of the position of the hand seen through prisms, but must be in the "judgment of the direction of the gaze." 2

Until very recent times, this conclusion was widely accepted. In 1963, however, Harris reported an experiment in which the adaptive effect, instead of transferring intermanually, was confined to the hand which $S$ had used for reaching. Prism adaptation, as it appeared in Harris' experiment, was a proprioceptive phenom- enon: a change in the judged position of the hand seen through prisms.

In 1964, Hamilton presented evidence that the adaptive effect transfers from exposed hand to unexposed hand when Ss are allowed to move their heads during adaptive exposure (as in Helmholtz' demonstration), but not when head movements are restricted (as was the case in Harris' experiment). Subsequent experiments (McLaughlin \& Bower, 1965a, b) failed to confirm this result; but Hamilton's paper nevertheless pointed the way to an explanation of the conflict in findings: depending on the conditions of adaptive exposure, the result may be a change in the judgment of the direction of gaze, a proprioceptive change associated with the hand and arm seen through prisms, or some combination of the two.

Each of these two kinds of adaptive change involves an alteration in the judged position of a part of the body. However, in the present paper, the term "proprioceptive change" will be reserved for the change in judged position of a part of the body seen through prisms, and the term "oculomotor change" will be used to designate a change in the judgment of the direction of gaze. This usage reflects the fact that the oculomotor change cannot be proprioceptive in the usual meaning of that term, for the eye lacks conscious position sense (Merton, 1964).

The present experiment was concerned primarily with the oculomotor type of prism-adaptive change. In particular, the aim was to identify the conditions of adaptive exposure which give rise to oculomotor change. Preliminary experiments had indicated that oculomotor change occurred when Ss simply looked at a complex visual display through prisms, with no part of the body seen: this exposure condition was therefore included in the experimental design. On the hypothesis that gross changes of fixation across the visual field seen through prisms should provide additional cues to adaptation, the second condition of adaptive exposure was identical to the first except that $S$ looked back and forth across the visual display repeatedly. In addition, two control conditions were used: one in which $\mathrm{S}$ saw (through prisms) his hand as well as the visual display; and another in which he saw nothing except the luminous target.

\section{Method}

The measure of the degree to which $\mathrm{S}$ is adapted to prisms is the accuracy with which he reaches or points (hand not seen) toward a visual target viewed 
through the prisms. It follows that the measure of adaptive change is the shift from inaccurate reaching (when $\mathrm{S}$ first looks through the prisms) to accurate reading (after he has become adapted).

There are several ways of analyzing the total adaptive change into its two components-for example, by determining the degree to which the adaptive change transfers to the un-adapted hand (Helmholtz, 1867; Hamilton, 1963), or by monitoring eye position in such a way as to measure any change in the judgment of the direction of gaze (McLaughlin \& Webster, in preparation). The method used in the present experiment was to have $S$ make manual judgments of "straight ahead" as well as of the position of the visual target (both judgments made with hand not seen). Shifts in "visual target" judgments provided a measure of total adaptive change; shifts in "straight ahead" judgments provided a measure of proprioceptive change (Harris, 1963; McLaughlin \& Bower, 1965b); and the amount of total adaptive change not accounted for by the proprioceptive change was taken as the measure of oculomotor change.

Subjects. Subjects were 12 college students, eight male and four female.

Apparatus. The visual target was a luminous white line $3 \mathrm{~cm}$ high and $0.2 \mathrm{~cm}$ wide. The $S$ viewed it from a distance of $33 \mathrm{~cm}$, his head being held in position by a bite plate bearing his dental impression. When making the dental impression, $\mathrm{S}$ was instructed to position himself so that the visual target (seen without prisms) appeared to him to be precisely straight ahead with respect to his head and body.

Directly beneath the target was a pointer which $\mathrm{S}$ could move along a horizontal track perpendicular to his line of sight. The pointer was tapered upward, its tip being $0.2 \mathrm{~cm}$ lower than the bottom edge of the visual target. Pointer, track, and visual target were mounted on a vertical plywood panel.

The visual display was illuminated by two light bulbs, one on each side of the display, which were shielded from S's direct view. With these lights off, $S$ could see only the visual target. When the lights were turned on, he could see, in addition, the pointer and his right hand. Other objects in the room were shielded from S's view, so that he could not see them even with the side lights on.

The prisms which were used (one before each eye) produced an angular deviation of approximately 11 degrees toward S's right.

All pointer settings were made with $S$ 's right hand, his left hand being kept out of his field of view. The $S$ signalled the completion of each pointer setting by tapping the table with his left hand.

Procedure. First, in total darkness (visual target off), $\mathrm{S}$ was instructed to set the pointer straight ahead. The visual target was then turned on, and S was again instructed to set the pointer straight ahead. For his third setting (still in the dark, neither hand nor pointer visible), $\mathrm{S}$ was instructed to set the pointer so that its tip was directly beneath the line. Following these three settings, $S$ was given a 5-sec, adaptive exposure. The second and third settings, and the adaptive exposure, were repeated 15 times, except that the final adaptive exposure was omitted: a total of 33 settings.

During a single experimental period, $\mathrm{S}$ adapted four times in succession, each time with a different condition of adaptive exposure. In Condition $A, S$ merely looked at the visual target in the dark. In Condition $\mathrm{B}$, he removed his hand from the pointer, the pointer was set directly beneath the line, the room lights were turned on (enabling him to see the pointer and the plywood panel), and he was instructed to fixate the tip of the pointer. Condition $\mathrm{C}$ was similar to Condition $B$ except that the pointer was moved back and forth at a uniform rate while $S$ fixated its tip. It was moved from the center position $10 \mathrm{~cm}$ toward S's right, back to center, $10 \mathrm{~cm}$ toward S's left, and back to center again. In Condition $D, S$ kept his hand on the pointer, the side lights were turned on (enabling him to see his hand and the pointer as well as the visual target), and he set the pointer directly beneath the target. In the first three conditions, $S$ did not see any part of his body; in the fourth condition, he saw only his right hand. The sequence of adaptation conditions was varied from $S$ to $S$ in such a way that each condition occurred at least twice in each of the four positions in an experimental session.

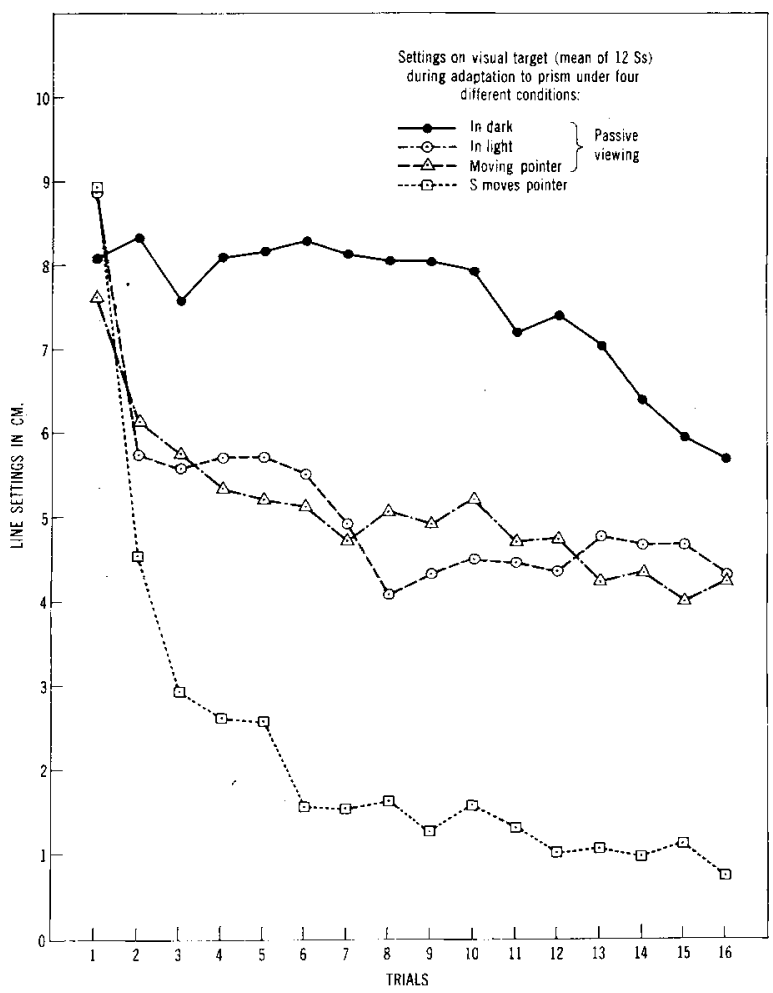

Fig. 1. Pointer settings on the visual target (mean data for 12 Ss) during adaptation to lateral prismatic displacement under each of four different conditions of adaptive exposure. 


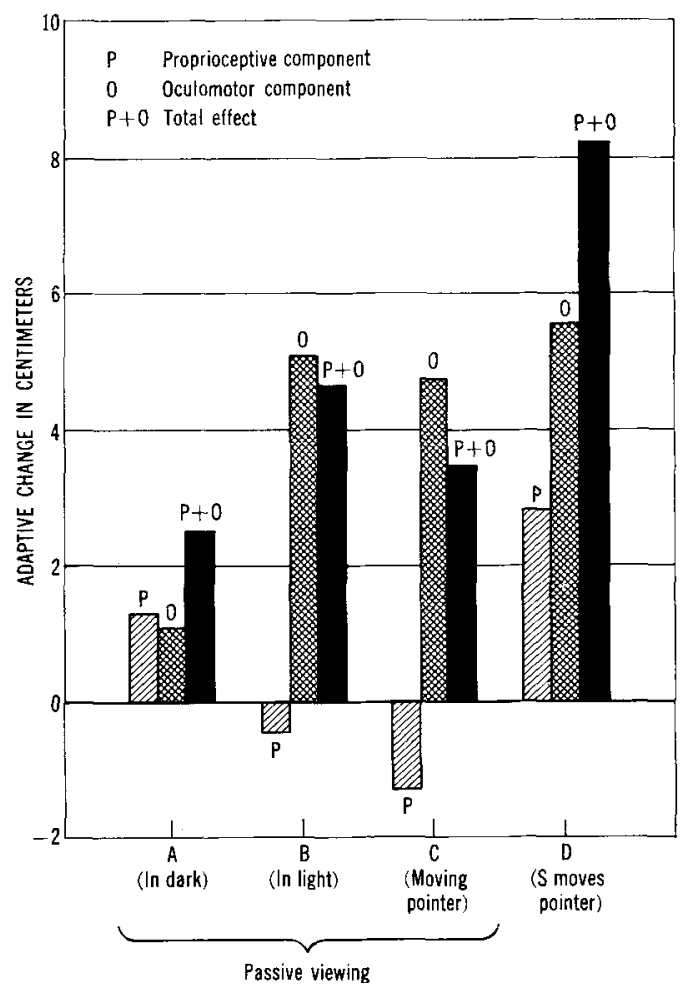

CONDITION OF ADAPTATION

Fig. 2. The total adaptive change analyzed into its two components (oculomotor and proprioceptive) for each of the four conditions of adaptive exposure.

Prior to each of the four adaptation periods in a single experimental session, $\mathrm{S}$ had 3-5 min. of hand-eye activity with prisms off, then approximately $30 \mathrm{sec}$. of adaptive exposure (hand seen through prisms) to prismatic deviation toward his left, and finally $1 \mathrm{~min}$. of hand-eye activity with prisms off.

\section{Results}

Total adaptive change. Figure 1 shows the course of adaptation under each of the four conditions ("visual target" settings only, mean results for $12 \mathrm{Ss})$. Some adaptation occurred even in total darkness (Condition A); and, when $\mathrm{S}$ was able to see his hand (Condition D), adaptation was nearly complete by the 6 th trial. The two conditions which involved viewing of the illuminated display with hand not seen produced an intermediate degree of adaptation. By analysis of variance, Conditions $B$ and $C$ did not differ significantly at $p>.05$; whereas all other inter-condition differences are significant at $\mathrm{p}<.01$.

Oculomotor and proprioceptive components.

The "straight ahead" judgments were used to analyze the total adaptive change into its two components. The shift in "straight ahead" settings, along with a numerically equal portion of the shift in "visual target" settings, was attributed to proprioceptive change; and the remain- ing portion of the shift in "visual target" settings was taken as the measure of oculomotor change.

This analysis, applied to the mean data for $12 \mathrm{Ss}$ (Fig. 2), reveals that viewing the visual target through prisms (Condition A) produced small and approximately equal amounts of oculomotor and proprioceptive change. Analysis of variance shows that there is a significant difference between the initial and terminal settings, such that both types of adaptive change are significant at $\mathrm{p}<.01$.

Conditions $\mathrm{B}$ and $\mathrm{C}$ produced a positive (i.e., an adaptive) visual change, and a negative (non-adaptive) proprioceptive change. Both types of change, for both conditions, are significant at $\mathrm{p}<.01$.

Adaptation with hand seen (Condition D) produced both oculomotor and proprioceptive change, both significant at $\mathrm{p}<.01$.

\section{Discussion}

Rapid adaptation with little adaptive exposure. One thing to be accounted for in these results is the large amount of adaptation (some $90 \%$ of the prism deviation under Condition D) after only $75 \mathrm{sec}$. of adaptive exposure. This is compatible with Helmholtz' report of reasonably complete adaptation after a brief exposure period; but it is entirely out of line with the results obtained by more recent experimenters. Held and Bossom, for example, obtained only 10\% adaptation after $2 \mathrm{hr}$. exposure, and their ss required four days of exposure to achieve $100 \%$ adaptation $(1961$, p. 35$)$.

It is likely that this difference in results is due to a difference in technique of measurement. In the present experiment, measurements of $S^{\prime} S$ adaptive state were made while $S$ was looking through the prisms. In contrast to this is the "after-effect" technique introduced by Held and Gottlieb in 1958 and widely used since then. The Held-Gottlieb procedure consists of recording S's sensory-motor responses before and after adaptive exposure, all measures being made without prisms. There is abundant evidence that the difference between "before" and "after" measures is smaller than the adaptive change as measured directly (see, for example, Wallach, Kravitz, \& Lindauer, 1963; Kohler, 1964, p. 97; Weinstein, Sersen, Fisher, \& Weisinger, 1964; Hamilton, 1964). In fact, Taylor (1964, p. 204) has described a well-practiced $\mathrm{S}$ who showed complete adaptation to prisms with no after-effect at all.

As further justification for the method of measurement employed here, two additional points maybe mentioned:

(1) Measurements made with the prisms in place provide a valid measure of prism adaptation as a normative process (Bevan, 1965): a shift from inaccurate reaching to accurate reaching. By contrast, the aftereffect procedure measures a non-adaptive shift from accurate reaching (without prisms, before adaptation) to inaccurate reaching (without prisms, after adaptation).

(2) By taking measures with the prisms in place, it is 
an easy matter to follow the time-course of adaptation. This facilitates the study of the adaptive process as an event over time rather than as a "before" and "after" phenomenon.

The stimulus conditions which produce oculomotor change. The experiment reported here was performed in July, 1965, but was not reported at that time because we wanted additional confirmation that oculomotor change occurs with hand not seen. We have obtained the same effect in two subsequent experiments, and a similar result has been reported by Harris et al (1966).

In the present experiment, one stimulus to oculomotor adaptation was the perceptual asymmetry of the visual display which $\mathrm{S}$ saw through prisms: the luminous target, which was by far the most prominent feature of the display, was 11 degrees to right of center. This type of asymmetry causes a concomitant shift in visual straight ahead (Wapner, Werner, Bruell, \& Goldstein, 1953). Such a shift would produce a change in judgment of the direction of gaze, for it would cause $S$ to choose, as the point of straight ahead fixation, a point to the right of veridical straight ahead. This is an adaptive response, for it causes $S$ to choose as "straight ahead" a point closer to the visual target than he would otherwise have chosen. He therefore comes to perceive the visual target as being closer to straight ahead ("more nearly straight ahead") than before; and this is reflected in an increased accuracy of pointer settings on the visual target.

There is another feature of the visual display seen through prisms which may also give rise to an adaptive oculomotor response-namely, the apparent rotation of the vertical plywood panel about a vertical axis, so that its right edge appears closer to $S$ than its left edge. We now advance the hypothesis that there is a tendency for $S$ to choose, as his straight-ahead line of sight, a line of sight normal to the panel. If this hypothesis is correct, then the apparent rotation of the panel about a vertical axis will cause $S$ to choose, as the point of straight-ahead fixation, a point to the right of veridical straight ahead (see Fig. 3). Exactly as in the case of perceptual asymmetry, this shift in visual straight ahead leads to an adaptive oculomotor change.

Conditions $\mathrm{A}$ and $\mathrm{B}$ were identical except that, under Condition A, the panel was not illuminated, so that the rotation of the panel about a vertical axis was not present (or was greatly diminished) as a stimulus condition. The oculomotor change which occurred under Condition A $(1.1 \mathrm{~cm})$ may therefore be attributed to the "asymmetry" factor alone. Assuming that asymmetry had the same effect under Condition B as under Condition $A$, the oculomotor change which occurred under Condition $B(5.0 \mathrm{~cm})$ may be analyzed as follows: $1.1 \mathrm{~cm}$ $(22 \%)$ caused by asymmetry of the visual display, and the remaining $3.9 \mathrm{~cm}(78 \%)$ caused by apparent rotation of the panel about a vertical axis.

The uvulomotor change which occurred under Condition $D$, where $S$ saw his hand through the prisms, was

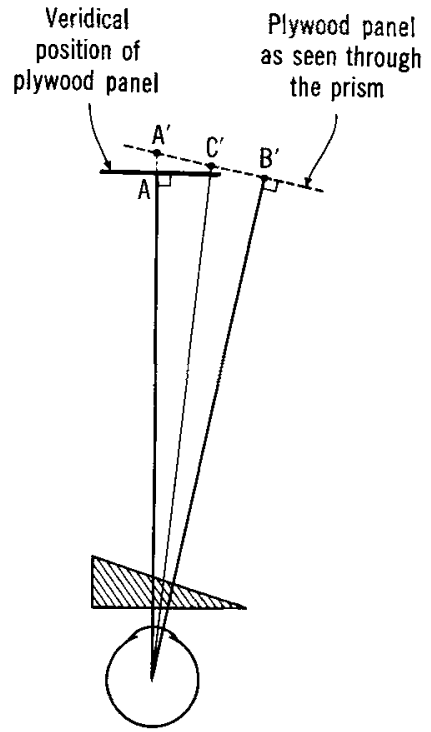

Fig. 3. Plan view showing the optical displacement of the plywood panel and its apparent rotation about the vertical axis. To simplify the explanation, monocular viewing rather than binocular viewing is illustrated. The point $A$ (seen without prism) is veridically straight ahead, and the line of sight to it is normal to the panel. With the prism in place, the point $A^{\prime}$ is veridically straight ahead, but $S^{\prime}$ 's line of sight is normal to the panel only at $B^{\prime}$. The hypothesis advanced here is that under these conditions $S$ will choose, as the straight ahead fixation point, some point $C^{\prime}$ between $A^{\prime}$ and $B^{\prime}$.

not significantly greater than that under Conditions B and $C$. This indicates that the oculomotor type of adaptive response is not facilitated when $S$ sees his hand through the prisms. Under the conditions of this experiment, then, oculomotor change (which acts to reduce hand-eye disparity) is caused entirely by secondary prismatic effects (asymmetry and rotation), and not by the hand-eye disparity itself.

Negative proprioceptive change. To account for the negative proprioceptive change which occurred under Conditions $\mathrm{B}$ and $\mathrm{C}$, it is necessary to use another untested hypothesis.

In the present experiment, with prism deviation toward S's right, an adaptive oculomotor change is a shift toward S's right. Such a change causes him to look toward his right (i.e., toward the displaced visual target) when attempting to look straight ahead. This in turn causes him to decide that the visual target is more nearly straight ahead, and his reaching behavior thus becomes more accurate.

In the same situation, however, any shift in reaching behavior toward S's right would be non-adaptive. The visual target (veridically straight ahead) appears to $S$ to be off to his right, and the only change in reaching behavior which will lead to more accurate responses is one which causes him to reach to the left of where he tries to reach.

To account for the non-adaptive proprioceptive change 
which occurred under Conditions $B$ and $C$, the following hypothesis is advanced: with hand not seen, an oculomotor change induces a shift in reaching behavior in the same direction as the oculomotor change, though not necessarily of the same magnitude.

This hypothesis seems to require ocular proprioception, contrary to the evidence cited by Merton (1964). However, the physiological requirements of the hypothesis can be met by a more conservative assumption, namely, that the pattern of efferent impulses to the ocular muscles is registered in the central nervous system. This pattern, when compared with the conscious direction of gaze, provides an index of the direction and extent of oculomotor adaptive change. It is therefore not inconceivable that the oculomotor adaptive change, even though not consciously perceived by $\mathrm{S}$, might induce a concomitant shift in reaching behavior.

Adaptive proprioceptive change under Condition A. The proprioceptive shift under Condition A was opposite in direction to that under Conditions $\mathrm{B}$ and $\mathrm{C}$. To interpret this result, it should be noted first that, under Condition $\mathrm{B}$, an oculomotor change of $5.0 \mathrm{~cm}$ induced a concomitant proprioceptive change of only $0.4 \mathrm{~cm}$. Assuming that the oculomotor change occurring under Condition A $(1.1 \mathrm{~cm})$ had an effect in proportion to its magnitude, it would induce a (non-adaptive) shift in reaching behavior of only $0.1 \mathrm{~cm}-\mathrm{a}$ negligible effect. What has to be accounted for under Condition A, then, is not the absence of a non-adaptive proprioceptive shift, but the presence of an adaptive proprioceptive shift of $1.3 \mathrm{~cm}$.

If this $1.3 \mathrm{~cm}$ shift was present under Condition $\mathrm{A}$, it may have been present under the other three experimental conditions as well, though counteracted or masked by effects of greater magnitude. The stimulus condition responsible for this shift, then, need not be peculiar to Condition $\mathrm{A}$, but may have been characteristic of all four experimental conditions.

We now advance the hypothesis that S's awareness of the veridical position of the visual target is enough to produce a small adaptive shift in reaching behavior. If this hypothesis is correct, then it may be that the small adaptive proprioceptive shift in Condition $A$ was a cognitive effect resulting from S's viewing of the visual target without prisms while making the dental impression.

Adaptation with hand seen. The results for Condition $D$ show that if, in addition to merely looking at an illuminated visual display through the prisms, $\mathrm{S}$ brings his hand into the field of view and carries out voluntary activity directed toward the target, then there occurs, in addition to the oculomotor change, a proprioceptive change.

This finding appears to be in conflict with the report by Hay and Pick (1966) that whole-body exposure (as contrasted with hand-only exposure) elicits oculomotor adaptation. That is, our finding seems to imply that the more of his body s sees throughprisms, the greater will be the proportion of proprioceptive to oculomotor change; whereas the Hay and Pick finding suggests that this is not the case. However, an oculomotor change is a change in the judgment of the position of the eyes with respect to the rest of the body; and, as already noted, oculomotor and proprioceptive changes are opposite in direction when both are adaptive. Oculomotor adaptation is therefore operationally indistinguishable from whole-body proprioceptive adaptation; and it may be that Hay and Pick's whole-body adaptive exposure produced whole-body proprioceptive adaptation.

Using the mean data for $12 \mathrm{Ss}$ in our experiment, the way in which the two types of adaptive change combined in Condition $\mathrm{D}$ may now be described.

At the beginning of the experiment, before any adaptation had taken place, $S$ perceived the visual target to be $7.6 \mathrm{~cm}$ off to the side (toward his right). At the end of the experiment, the oculomotor change caused him to choose, as the point of straight-ahead visual fixation, a point $5.4 \mathrm{~cm}$ to the right of his initial straight-ahead judgment. As a result, he judged the target to be $5.4 \mathrm{~cm}$ closer to straight ahead than before-i.e., only $2.2 \mathrm{~cm}$ from straight ahead.

The subject now tries to set the pointer $2.2 \mathrm{~cm}$ to right of center (hand not seen). At the start of the experiment, he could have done this; but he has now acquired a proprioceptive error such that his hand (not seen) is $1.5 \mathrm{~cm}$ to the left of where he thinks it is. (The proprioceptive shift of $2.8 \mathrm{~cm}$ shown in Fig. 2 is the sum of (a) an error of $1.2 \mathrm{~cm}$ right in initial straight-ahead setting and (b) an error of $1.5 \mathrm{~cm}$ left in final straightahead setting). As a result, in trying to set the pointer at $2.2 \mathrm{~cm}$, he sets it instead at $0.7 \mathrm{~cm}$ : a more accurate setting.

Wooster's experiment and reafference. In 1923, Margret Wooster reported an extensive series of experiments in which Ss showed large adaptive changes in reaching behavior (pointing at a visual target seen through prisms) following prism-adaptive exposure with no part of the body seen. Harris (1965) explained this finding by pointing out that head movements were not restricted during adaptive exposure in Wooster's experiment; but Harris did not explain how adaptation might result from head movements only, in the absence of other adaptive cues; nor did he attempt to reconcile the contradiction between Wooster's results and the findings of more recent investigators regarding the role of reafferent stimulation in prism adaptation.

The hypotheses advanced above, regarding the effects of perceptual assymmetry and apparent rotation of the visual field in producing oculomotor change, are particularly well suited to the interpretation of Wooster's experiment. The prismatic deviation in Wooster's experiment was nearly twice as large as that used in the present experiment (21 degrees instead of 11 degrees), so that both assymmetry and rotation were more pronounced. Wooster's apparatus provided a textured curtain, perpendicular to $S$ 's line of sight, covering substantially the entire visual field, so that 
textural gradients (Gibson, 1950, p. $77 \mathrm{ff}$. ) would accentuate the "rotation" effect. The viewing distance (to the textured curtain) was quite short-on the order of $20 \mathrm{~cm}$ (exact distance not specified), which would further accentuate the perceptual effect of apparent rotation. With the curtain drawn back, the visual display seen through the prisms consisted chiefly of horizontal contours perpendicular to S's line of sightcontours which would provide the perspective needed to make the prismatic rotation strikingly evident to $\mathrm{S}$.

We suggest, therefore, that the adaptive changes in reaching behavior which occurred in Wooster's experiments represented an oculomotor change caused by the apparent rotation of the visual display about a vertical axis.

This interpretation suggests that those investigators who find that reafferent stimulation is essential to prism adaptation (for example, Hein \& Held, 1962) are dealing primarily with the proprioceptive type of adaptive change; whereas those investigators who find adaptation occurring in the absence of reafferent stimulation (Wallach, Kravitz, \& Lindauer, 1963; Weinstein, Sersen, Fisher, \& Weisinger, 1964; Howard, Craske, \& Templeton, 1965; Singer \& Day, 1966) are dealing primarily with oculomotor adaptation.

\section{Conclusions}

1. When $S$ sees through prisms only a luminous visual target in a dark surround, the result is a small adaptive proprioceptive change accompanied by a small adaptive oculomotor change. The proprioceptive change is attributed to a cognitive effect resulting from $S$ 'S previous knowledge of the veridical position of the visual target. The oculomotor change is attributed to the effect of assymmetry of the visual display seen through prisms.

2. When $\mathrm{S}$ sees through prisms the same visual target, but with an illuminated surround, the result is a large adaptive oculomotor change accompanied by a small proprioceptive change in the non-adaptive direction. The oculomotor change is attributed to the combined effects of (a) asymmetry and (b) apparent rotation about a vertical axis of the panel which formed the surround of the visual target. On the basis of the fact that the two changes (adaptive oculomotor and nonadaptive proprioceptive) are in the same direction, it is suggested that the proprioceptive shift which occurs under this type of adaptive exposure may be a secondary effect, induced by the oculomotor change.

3. The oculomotor type of adaptive change is not enhanced by oculomotor activity.

4. When $S$ sees through prisms his hand as well as a luminous target with illuminated surround, the result is (a) an oculomotor change equal in magnitude to that which occurs with hand not seen, and (b) a change in the judged position of the hand seen through prisms. The two effects combine additively to produce nearly complete adaptation to prisms following only $75 \mathrm{sec}$. of adaptive exposure.

5. The hypotheses advanced here to account for the occurrence of oculomotor adaptive change with hand not seen are also capable of accounting for the previously unexplained results reported by Wooster in 1923 .

6 . The current controversy about the role of reafferent stimulation in prism adaptation may result from the failure of some investigators to distinguish between the oculomotor and proprioceptive types of adaptive change. It may be that those investigators who find reafference essential for adaptation to prismatic displacement are dealing primarily with proprioceptive adaptive changes, whereas those investigators who find adaptation occurring without reafference are dealing primarily with oculomotor adaptive changes.

\section{References}

Bevan, W. The concept of adaptation in modern psychology. $J$ Psychol., 1965, 59, 73-93.

Gibson, James J. The perception of the visual world. Boston: Houghton Mifflin Company, 1950.

Hamilton, C. R. Intermanual transfer of adaptation to prisms. Amer. J. Psychol., 1964, 77, 457-462.

Harris, C. S. Adaptation to displaced vision: visual, motor, or proprioceptive change? Science, 1963, 140, 812-813.

Harris, C. S. Perceptual adaptation to inverted, reversed, and displaced vision. Psychol. Rev., 1965, 72, 419-444.

Harris, C. S., Harris, J., \& Karsch, C. Shifts in pointing straight ahead after adaptation to prisms. Paper presented at Eastern Psychological Association, New York, April, 1966.

Hay, J. C., \& Pick, H. L. Visual and proprioceptive adaptation to optical displacement of the visual stimulus. J. exp. Psychol., $1966,71,150-158$.

Hein, A., \& Held, R. A neural model for labile sensorimotor coordinations. Biological Prototypes and Synthetic Systems. Vol. 1. Now York: Plenum Press. Pp. 71-74.

Held, R., \& Bossom, J. Neonatal deprivation and adult rearrangement: complementary techniques for analyzing plastic sensorymotor coordinations. J. comp. physiol. Psychol., 1961, 54, $33-37$.

Held, R., \& Gottlieb, N. Technique for studying adaptation to disarranged hand-eye coordination. Percept. mot. Skills, 1958, $8,83-86$.

Helmholtz, H. von. Handbuch der Physiologischen Optik, Leipzig: Leopold Voss, 1867.

Howard, I., Craske, B., \& Templeton, W. B. Visuomotor adaptation to discordant exafferent stimulation. J. exp. Psychol., 1965, 70, 189-191.

Kohler, I. The formation and transformation of the visuai world. (Trans. by H. Fiss) Psychol. Issues, 1964, $3(\dot{4})$.

McLaughlin, S. C., \& Bower, J. L. Auditóry localization and judgments of straight ahead during adaptation to prism. Psychon. Sci., 1965a, 2, 283-284.

McLaughlin, S. C., \& Bower, J. L. Selective intermanual transfer of adaptive effects during adaptation to prism. Psychon. Sci. $1965 \mathrm{~b}, 3,69-70$.

McLaughlin, S. C., \& Webster, R. G. Oculomotor change during adaptation to displaced vision. In preparation, 1966.

Merton, P. A. Absence of conscious position sense in the human eyes. In M. B. Bender (Ed.), The oculomotor system. New York: Harper \& Row, 1964. Chapter 14.

Singer, G., \& Day, R. H. Spatial adaptation and after-effect with opticaliy transformed vision: effects of active and passive responding and the relationship between test and exposure responses. J. exp. Psychol., 1966, 71, 725-731. 
Taylor, J. G. The behavioral basis of perception. New Haven: Yale University Press, 1962.

Wallach, H., Kravitz, J. H., \& Lindauer, Judith. A passive condition for rapid adaptation to displaced visual direction. Amer. J. Psychol, 1963, 76, 568-578.

Wapner, S., Werner, H., Bruell, J. H., \& Goldstein, A. G. Experiments on sensory-tonic field theory of perception: VII. effect of asymmetrical extent and starting positions of figures on the visual apparent median plane. J. exp. Psychol., 1953, 46. 300-307.

Weinstein, S., Sersen, E. A., Fisher, L., \& Weisinger, M. Is reafference necessary for visual adaptation? Percept. mot. Skills, $1964,18,641-648$.

Wooster, Margaret. Certain factors in the development of a new spatial coordination. Psychol. Monogr., 1923, 43(4), 1-96 (Whole No. 146).

\section{Notes}

1. The first edition of von Helmholtz' Handbuch is cited here to , give an accurate date. The quotations are from J. P. C. Southall's translation of the third edition (The Optical Society of America, 1924). The description of prism adaptation in the third edition (Vol. 3, p. $246 \mathrm{ff}$,) does not differ from that in the first edition. (p. $601 \mathrm{ff}$.)

2. The phrase "judgment of the direction of the gaze" is Southall's translation of "die Beurtheilung der Blickrichtung."

3. The research described here was supported in part by Contract MD-2714 with the U. S. Army Medical Research and Development Command, and in part by Research Grant GB-4196 from the National Seience Foundation.

(Accepted for publication October 19, 1966.) 